\title{
Competencies for Entry into the Profession of Dental Hygiene
}

\author{
Soo-Myoung Bae, Won-Gyun Chung ${ }^{1}$, Jong-Hwa Jang ${ }^{2}$, So-Jung Mun ${ }^{1}$, \\ Bo-Mi Shin, and Sun-Jung Shin ${ }^{\dagger}$ \\ Department of Dental Hygiene, College of Dentistry and Research Institute of Oral Science, Gangneung-Wonju National \\ University, Gangneung 25457, \\ ${ }^{1}$ Department of Dental Hygiene, Yonsei University Wonju College of Medicine, Wonju 26426, \\ ${ }^{2}$ Department of Dental Hygiene, Hanseo University, Seosan 31962, Korea
}

This study was conducted to derive core and detailed competencies of dental hygienists to be utilized as educational targets to be reached by graduation as well as basic data that can be reflected in the development and improvement of dental hygiene curricula. This study analyzed publication reports from the Korean Dental Hygienists Association, the International Federation of Dental Hygienists, the Commission on Dental Accreditation, and the American Dental Education Association. Based on the academic classification system for dental hygiene studies, the components of core and detailed competencies of dental hygiene school at the time of graduation were extracted and developed through expert panel discussions. This study defined competencies at the graduation level of dental hygiene school and derived eight core competencies and their 52 detailed competencies to serve as educational objectives from four areas: professionalism, communication, clinical practice, and community and health promotion. In the future, it will be necessary to conduct self-assessments of competencies based on those developed in this study, at time of the graduation from dental hygiene school, as well as to continuously develop competency-based curricula according to entry level, knowledge level, and graduation level. Thus, it is urgently necessary to develop a system that can evaluate the competencies of dental hygienists after graduation and put this system into practical use.

Key Words: Competency-based education, Core competency, Dental hygiene, Specific competency

\section{Introduction}

Competency is defined as the ability to complete a given task. It was first discussed as an academic topic by McClelland ${ }^{1)}$, who noted that human ability cannot be properly measured by traditional academic aptitude tests or intelligence tests, and proposed studying 'competency' as a method of predicting work outcomes by examining the characteristics of successful people and the spontaneous behaviors that were the source of their success. Subsequently, Spencer and Spencer ${ }^{2}$ refined the concept of competency as inherent characteristics of the individual that enable them to complete an appropriate task or job with excellence in a given profession or situation. In the field of nursing, competency includes aspects such as the ability to integrate knowledge in actual work, experience, critical thinking, technical skill, care, communication, environment, motivation, and professionalism ${ }^{3}$. The Korean Accreditation Board of Nursing emphasizes on a competency-based curriculum to improve the competency of nurses and the quality of nursing education; it presents an important framework for 2-week accreditation, setting competency-based education goals at each college of nursing, striving to improve curricula and assessment methods, compiling competency profiles for the professional roles, defining required knowledge, technique, and 
attitude, and improving curricula and reinventing educational methods based on this evidence ${ }^{4)}$. Similarly, the College and Graduate College of Dentistry Accreditation Criteria are one standard of accreditation that present methods of assessment for curriculum outcomes and suggest competencies for dentists at the national level, as well as detailed competencies for individual fields ${ }^{5)}$. Thus, examinations for issuance, receipt, and renewal of professional licenses, permits, and qualifications are also being readjusted in accordance with competencies.

Following 50 years of quantitative growth at higher education institutions, educational institutions for dental hygiene in Korea are focusing their efforts to consistently produce high-quality academic and educational systems ${ }^{6}$. Choi et al. ${ }^{7)}$ argued for the need to unify and standardize the competencies of Korean dental hygienists through comparison with entry-level academic competencies in the United States, based on the standard competencies for dental hygienists presented by the American Dental Education Association (ADEA). Kim et al. ${ }^{8)}$ recently explored the competencies required by Korean dental hygienists, suggesting eight detailed competencies for national-level dental hygienists: integrating academic knowledge, professionalism, communication, cooperation, health improvement, oral health education, patient management, and administration. However, there are many tasks that still need to be accomplished, such as investigating competencies at the time of graduation from a given curriculum, developing standardized curriculum a based on these competencies, educating learners with the methods required to help them achieve these competencies, and evaluating their achievement of these competencies.

In order to improve the quality of dental hygiene education, it is important to strengthen the core competencies demanded of dental hygienists, and to make these core competencies the basis of dental hygiene curricula. As public health professionals, dental hygienists are responsible for the oral health of the nation and their work has a major social impact. As such, defining their identity as public health professionals, examining the competencies they need to possess, and implementing education based on these competencies is a very important founda- tion for the overall quality of life of all members of society. Hence, the present study aims to provide a basic resource that can be reflected in future development and improvement of dental hygiene curricula. To this end, we reviewed the case of the United States, where research on the competencies of dental hygienists was first published, and, through discussion with an expert panel group, we identified core and detailed competencies that could serve as educational goals for dental hygienists to achieve by graduation.

\section{Materials and Methods}

1. Extracting core and detailed competencies for dental hygienists through an analysis of the domestic and international literature

We reviewed the definition and responsibilities of dental hygienists in Korea based on the domestic and international literature. Specifically, we examined dental hygienist competencies suggested by the Korean Dental Hygienists Association (KDHA), the International Federation of Dental Hygienists (IFDH), the U.S. Commission on Dental Accreditation (CODA) ${ }^{9}$, who presented dental hygiene education accreditation criteria in 2012, and the $\mathrm{ADEA}^{10)}$. Five researchers stipulated core competencies centered on the definition and responsibilities of dental hygienists and the core and detailed competencies for dental hygiene at the time of graduation were extracted by domain.

\section{Development of core and detailed dental hygiene} competencies through an expert panel discussion

We evaluated the terminology and content validity of the core competencies by domain, and the content and validity of the detailed competencies. To this end, we held an expert panel discussion with the Chairperson of the Korean Association of Dental Hygiene Professors, the Chairperson of the Korean Society of Dental Hygiene Science, two professors of dental hygiene, and two collaborating researchers. The terminology and domains of the suggested core competencies were defined, and their suitability was evaluated. The relevance to work, timeliness, and number of the detailed competencies 
Table 1. Compared of Competencies for Dental Hygiene

\begin{tabular}{|c|c|c|}
\hline Classification & Category of competencies & Key word of competencies \\
\hline \multirow[t]{8}{*}{ CODA } & Patient care competencies & Dental hygiene care \\
\hline & & Communication skills \\
\hline & & Health promotion \\
\hline & & Disease prevention activities \\
\hline & Ethics and professionalism & Ethical decision making \\
\hline & & Professional responsibility \\
\hline & Critical thinking & Self-assessment skills \\
\hline & & Life-long learning \\
\hline \multirow[t]{23}{*}{ ADEA } & Core competencies & Ethics \\
\hline & & Critical thinking skills \\
\hline & & Comprehensive problem-solving \\
\hline & & Responsibility for professional actions \\
\hline & & Self-assessment skills \\
\hline & & Communication skills \\
\hline & & Providers for optimal patient care \\
\hline & Health promotion and disease prevention & Health promotion \\
\hline & & Promote health-related quality of life \\
\hline & & Disease prevention or health maintenance strategies \\
\hline & Community involvement & Health care system \\
\hline & & Community oral health service \\
\hline & & Patient's access to oral health service \\
\hline & & Community-based program \\
\hline & & Advocate \\
\hline & Patient/client care & Dental hygiene care \\
\hline & & Assessment \\
\hline & & Dental hygiene diagnosis \\
\hline & & Planning \\
\hline & & Implementation \\
\hline & & Evaluation \\
\hline & Professional growth and development & Professional goal \\
\hline & & Social network \\
\hline \multirow[t]{27}{*}{ KOREA } & Professional conduct & Ethical decision making \\
\hline & & Professional behavior \\
\hline & & Self-assessment skills \\
\hline & & Life log learning \\
\hline & & Accumulate evidence \\
\hline & Ethical decision making & Ethical decision making \\
\hline & & Apply Ethical principle \\
\hline & Evidence based decision making and problem solving & Dental hygiene research \\
\hline & & Evidence based dental hygiene \\
\hline & Communication & Understanding psychological development \\
\hline & & Effective communication \\
\hline & Clinical dental hygiene & Dental hygiene care \\
\hline & & Assessment \\
\hline & & Dental hygiene diagnosis \\
\hline & & Planning \\
\hline & & Implementation \\
\hline & & Evaluation \\
\hline & Clinical practice & Anxiety and dental fear care \\
\hline & & Understanding dental radiography \\
\hline & & Perform tasks for effective dental service \\
\hline & & Patient management \\
\hline & Hospital administration & Utilize document \\
\hline & & Claim dental insurance \\
\hline & & Leadership \\
\hline & Community and health promotion & Community oral health service \\
\hline & & Planning community-based program \\
\hline & & Evaluating community-based program \\
\hline
\end{tabular}

CODA: Commission on Dental Accreditation, ADEA: American Dental Education Association. 
suggested for each domain was reviewed, and their suitability was evaluated.

\section{Results}

\section{Comparison with competencies of U.S. dental hygienists}

CODA presented accreditation criteria for dental hygiene education, in which they suggested specific classes that need to be included in curricula within four scopes: general studies, biomedical science, dentistry, and dental hygiene. The detailed competencies required by graduates in dental hygiene were dental hygiene management, ethical thinking, professional behavior, and critical thinking.

ADEA categorized the competencies required by new dental hygienists into essential core competencies, health improvement, disease prevention, community participation, application of dental hygiene management, effort to grow as a professional, and self-development. Core competencies included proper attitude as a dental hygienist, ethical awareness, and communication. Detailed competencies were presented for each core competency.

In Korea, orized the competencies required by new dental hygienists into essential core compet the oral health of the public by supporting oral health education, preventative dental treatment, dental care assistance, and administration. Specific roles for dental hygienists are oral health promotion and education, education/researcher, preventative dental practitioner, dental care assistant, and hospital administrator ${ }^{11}$. IFDH defined 'dental hygienist' as a healthcare professional who helps subjects maintain optimal oral health by preventing and treating oral diseases through processes such as clinical service and education, establishment of consulting plans, and assessment. Thus, it can be said that dental hygienists should at least possess comprehensive professional clinical ability, including the appropriate knowledge, technique, and attitude in clinical dentistry and dental hygiene management at the time of graduation. Moreover, in order to properly promote oral health and prevent disease, dental hygienists need to meet the competencies of evidence-based decision making, problem solving, professional mentality, ethical decision making, proper communication, and leadership (Table 1).

\section{Core and detailed competencies for dental hygiene as education goals by domain}

'Professionalism', 'communication', 'clinical', and 'health improvement and the local community' were identified as domains of dental hygiene at the time of graduation. A total of eight core competencies were suggested for each domain: professional behavior, ethical decision making, evidence-based decision making and problem solving, communication, clinical dental hygiene, clinical dentistry, hospital administration, health improvement and the local community. In addition, a total of 52 detailed competences were identified that could be set as educational goals to help achieve the core competencies, including 15 detailed competencies in the professionalism domain, two in the communication domain, 28 in the clinical domain, and seven in the health improvement and the local community domain. Looking at the detailed contents of the core competencies, dental hygienists graduating from some colleges are required to have critical thinking and the latest clinical knowledge (professional behavior), and some are required to fulfill and have knowledge about their moral and ethical responsibility for health guidance to patients in the treatment room and to the general public (ethical decision making). Some graduates are required to have the competency to communicate and interact effectively with various populations, such as patients, patients' families, colleagues, and public health specialists (communication). Some need to be able to perform dental hygiene management under the guidance of the patient/subject's dental hygienist (clinical dental hygiene), and some need to possess the knowledge and technique for basic clinical work that is essential to the responsibilities of a dental hygienist (clinical dentistry). Others need to be competent in document and hospital management (hospital administration), and some need to ascertain the demand for oral health as part of local projects to improve health and prevent disease, and to plan, implement, and assess oral health projects (health improvement and the local community). Table 2 displays the detailed competencies for each of the core competencies. 


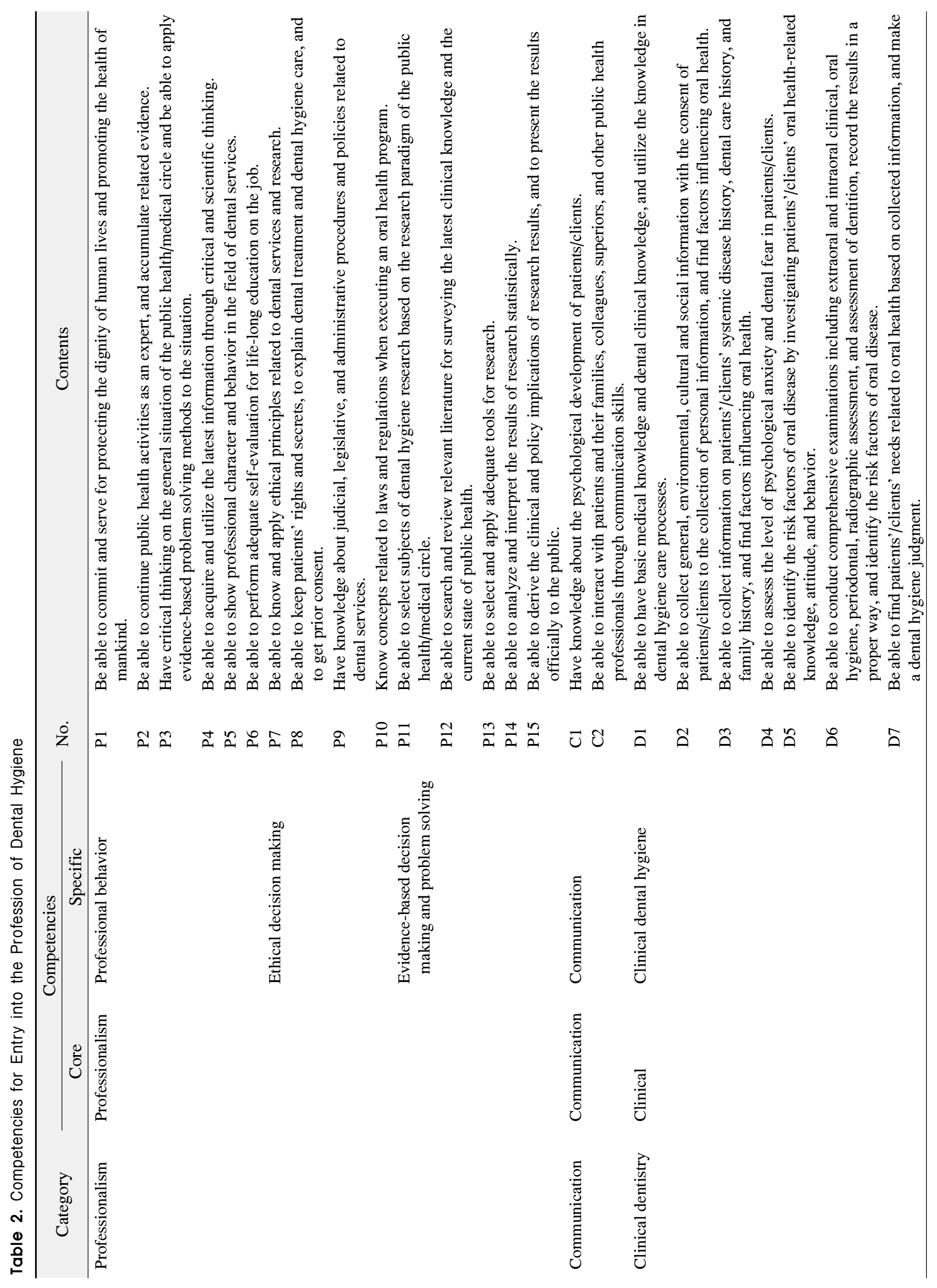




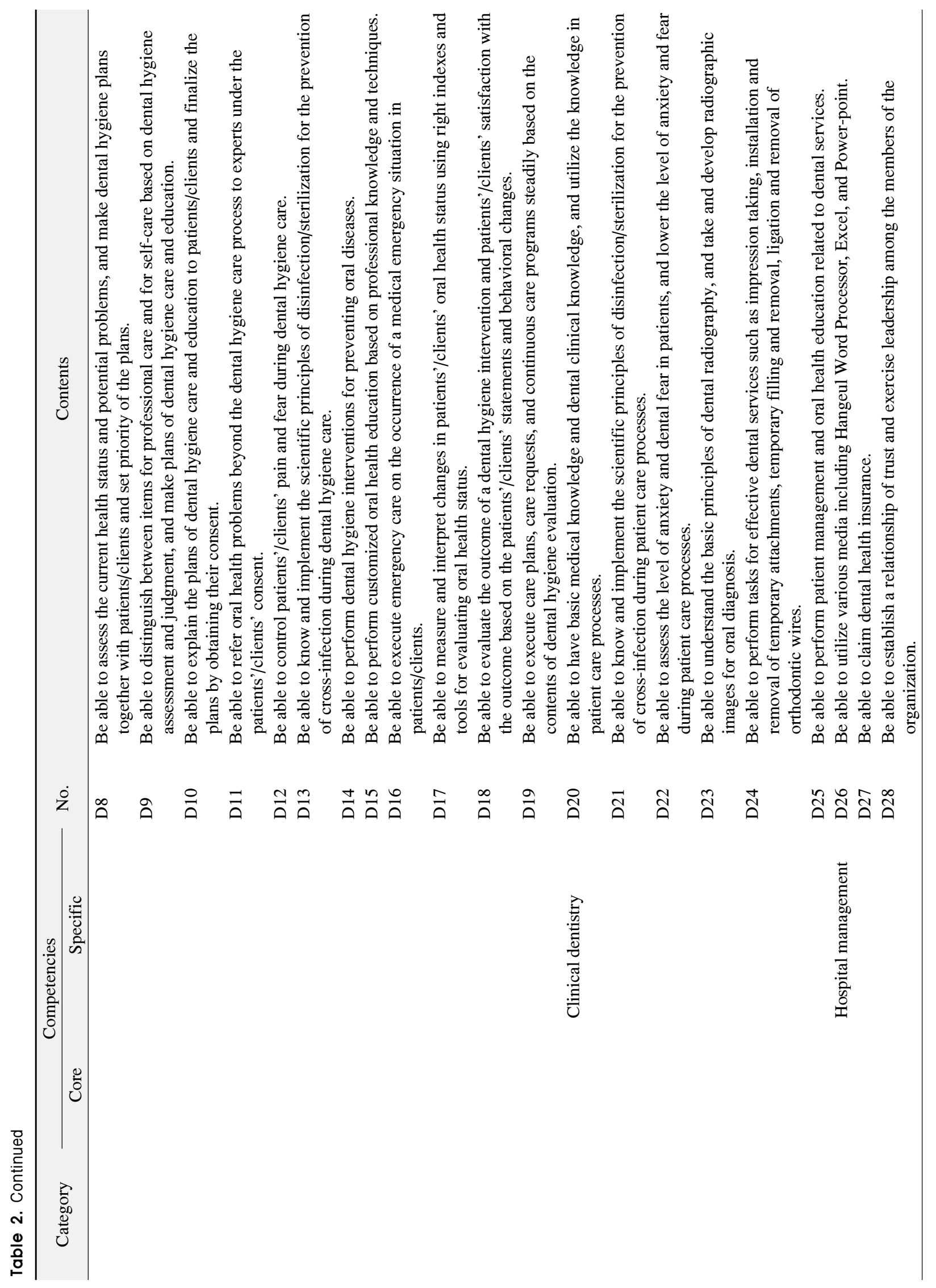




\section{Discussion}

Recently, particularly in medical and dental education, the need to develop task-based competencies from conventional knowledge-based learning goals has been highlighted. At the same time, efforts are being made to set competency-based educational goals and to develop and implement curricula that allow competencies to be calculated as learning outcomes ${ }^{12,13)}$. In the present study, we deduced core and detailed competencies as educational goals for dental hygienists in college, with the aim of providing basic information that can be used in the development and improvement of future dental hygiene curricula.

We were able to present required competencies for dental hygienists at the time of graduation based on the educational goals of various colleges. Using this method, we identified eight core competencies, by domain: professional behavior, ethical decision making, evidence-based decision making and problem solving, communication, clinical dental hygiene, clinical dentistry, hospital administration, and health improvement and the local community. Fifty-two detailed competencies were identified and categorized in order to aid the core competency goal achievement. The core competencies presented in this study show some similarities with the competencies presented by CODA and ADEA in most domains. By developing competencies based on the role of dental hygienists and the classification system of dental hygiene studies in Korea, we provide competencies that enable linkage between theory and practical work.

New dental hygienists take courses like clinical practice, but they experience limitations in performing independent clinical work immediately after graduation. Typically, they will spend a $1 \sim 3$ month period of re-education, training under a senior hygienist ${ }^{14)}$. This is very similar in nursing, where new nurses receive separate education because their work performance does not meet the demands of patients or the work environment. In order to reduce the time and costs of this additional education and to improve new nurses' ability to adapt to real working environments, some authors have argued for the need to reflect such demand sand real work scenarios in 
nursing curricula ${ }^{15)}$. Consequently, the Korean Accreditation Board of Nursing has improved the quality of nursing education by continually revising education programs to produce students with the competencies demanded in domestic and international public healthcare. Currently, they are performing accreditation for nursing education and suggesting core nursing competencies at the time of graduation ${ }^{4}$. In the field of dental hygiene, the Committee to Advance Establishment of a Korean Dental Hygiene Education Accreditation Institute was for 2010. Following the first public hearing in 2014, criteria for a Korean Dental Hygiene Education Accreditation Institute were shared at the second public hearing in December 2016, which increased interest in core competencies for dental hygiene to improve the quality of dental hygiene education. These will become important indices for preparing a balanced educational system based on cultivating professional competencies in dental hygienists. Competency-based curricula are expected to be able to minimize the discrepancy between the education and health care environments by increasing the proportion of dental hygienists achieving there quired competencies by graduation.

This study suggested core and detailed competencies that are applicable to some colleges of dental hygiene. However, one limitation is that these competencies do not necessarily reflect the actual level of competency in students currently enrolled at colleges of dental hygiene. Nevertheless, the core and detailed competencies developed in this study are presented as educational goals in dental hygiene that can provide a basis to explore measures to develop, expand, and systematize future competency-based curricula. Moreover, this study is significant because it can be used as basic information to suggest competencies for dental hygienists at the time of graduation, according to the educational goals at each college, and to develop measures to standardize curricula.

In order to calculate core and detailed competencies as academic outcomes at the time of graduation and to link these with curricula, we believe it will be necessary to construct an assessment system and develop assessment tools to appropriately evaluate achievement of competencies.

\section{Acknowledgements}

We express our gratitude to funding from Korean association of dental hygiene professors in 2014, which made it possible to complete this study.

\section{References}

1. McClelland DC: Testing for competence rather than for "intelligence". Am Psychol 28: 1-14, 1973.

2. Spencer LM, Spencer SM: Competence at work: models for superior performance. John Wiley \& Sons, New York, pp.9-16, 1993.

3. Smith SA: Nurse competence: a concept analysis. Int J Nurs Knowl 23: 172-182, 2012.

4. Korean Accreditation Board of Nursing Education: Evaluation criteria of nursing education certification (Bachelor of Nursing). Korean Accreditation Board of Nursing Education, Seoul, pp.3-38, 2012

5. Korean Institute of Dental Education and Evaluation (KIDEE): National competencies for Korean dentists. KIDEE, Seoul, pp.25-47, 2010.

6. Yoo JS, Kang KH, Kim YN, Park JR, Lee SM, Jang JH: A study on academic classification formulation of the dental hygiene discipline. Korean Association of Dental Hygiene Professors, Seoul, pp.67-73, 2014.

7. Choi DS, Kim SH, Kim JS: A comparative analysis of competencies in American dental education association and American dental hygiene schools. J Korean Soc Dent Hyg 15: 547-553, 2015.

8. Kim SH, Sharon MC, Minn NY, et al.: Development of national level dental hygienist competency. Korea Dental Hygienists Association, Seoul, pp.71-75, 2016.

9. Commission on Dental Accreditation: Accreditation standards for dental hygiene education programs. American Dental Association, Chicago, pp.12-44, 2013.

10. American Dental Education Association (ADEA): ADEA competencies for entry into the allied dental professions. ADEA, Washington, pp.1-12, 2011.

11. Korean Dental Hygienists Association: Introduction to dental hygienist. Retrieved April 4, 2017, from http://www. kdha.or.kr/introduce/dentalhygienist.aspx\#introduce(2016).

12. Park BY: A study on the concept and the pedagogical 
meaning of the competency-based dental education: based on the competency profile of ADEA. J Employ Skills Dev 11: 215-235, 2008.

13. Kim JA, Ko JK: Modeling core competencies in the competency-based nursing curriculum. J Korea Acad Indust Coop Soc 16: 7635-7647, 2015.
14. Kang YJ: Analysis on the recognition occupational work training in new dental hygienist. J Korean Soc Dent Hyg 7: 365-379, 2007.

15. Lee SK, Park SN, Jeong SH: Nursing core competencies needed in the fields of nursing practice for graduates in nursing. J Korean Nurs Adm Acad Soc 18: 460-473, 2012. 